\title{
Is there an association of socioeconomic deprivation with acute primary angle closure?
}

\author{
Edward Saxby $\mathbb{1 D}^{1} \cdot$ Kelvin Cheng $^{1} \cdot$ Niamh O'Connell $^{2} \cdot$ Roshini Sanders ${ }^{2,3} \cdot$ Pankaj Kumar Agarwal ${ }^{1}$
}

Received: 28 December 2020 / Revised: 14 May 2021 / Accepted: 19 May 2021 / Published online: 11 June 2021

(c) The Author(s), under exclusive licence to The Royal College of Ophthalmologists 2021

\begin{abstract}
Background Socioeconomic deprivation is known to increase the risk of late presentation of many diseases. This is the largest study in United Kingdom investigating the relationship between socioeconomic deprivation and acute primary angle closure (APAC).

Methods A retrospective review of case notes was conducted of 718 consecutive patients who underwent laser peripheral iridotomy (LPI) in Edinburgh (Princess Alexandra Eye Pavilion) and Fife (Queen Margaret Hospital) between 2015 and 2019. Baseline demographics including sex, age, ethnicity, pre-existing diabetes, use of anti-depressants, and family history of glaucoma were collected. Deprivation was scored using the Scottish Index of Multiple Deprivation (SIMD) Index 2020v2. A lower rank and decile indicate higher degrees of deprivation. We investigated differences in characteristics between patients who were referred routinely versus patients who referred as APAC.

Results The SIMD rank and deciles were consistently lower in patients who were referred urgently with APAC in both centres $(P=<0.05)$ when compared to those referred routinely for LPI. On univariate and multivariate logistic regression, the presentation of APAC is negatively associated with SIMD Decile ( $\mathrm{OR}=-0.101,95 \% \mathrm{CI}-0.178$ to $-0.026, P=0.008)$ and family history of glaucoma $(\mathrm{OR}=-1.010,95 \% \mathrm{CI}-1.670$ to $-0.426, P=0.001)$, and positively associated with age $(\mathrm{OR}=0.029,95 \%$ CI $0.009-0.049, P=0.004)$.

Conclusions Socioeconomic deprivation is an important risk factors for patients presenting with APAC. Socioeconomic deprivation should be incorporated into the design of glaucoma services and considered when triaging patients for prophylactic and therapeutic LPI and cataract surgery.
\end{abstract}

\section{Introduction}

Glaucoma remains the leading cause of irreversible blindness worldwide [1]. In the UK, despite widely available treatment, glaucoma is the second commonest cause of visual impairment following age-related macular degeneration [2]. During the COVID-19 era, glaucoma management has presented a particular challenge because of the largely asymptomatic nature of the disease and the requirement for detailed stereoscopic examination of intra-ocular structures,

Edward Saxby

Edward.saxby@nhslothian.scot.nhs.uk

1 Princess Alexandra Eye Pavilion, Edinburgh, UK

2 Ophthalmology Department, Queen Margaret Hospital, Dunfermline, Fife, UK

3 University of Edinburgh, Edinburgh, UK primarily gonioscopy. Pre pandemic one UK centre reported $86 \%$ of all glaucoma attendances involved a face-to-face visit with either a consultant or specialist optometrist. As with other outpatient specialities during the pandemic, many glaucoma clinic appointments have had to be postponed [3]. Patients are facing ever increasing waiting times. To ensure no patients come to harm, we as clinicians must recognise those within the glaucoma service and indeed the wider ophthalmic service that have significant risk factors for visual loss and triage accordingly.

In 2015 the Scottish intercollegiate guidelines network (SIGN) released its guidance for glaucoma referral and safe discharge [4]. Within this document was included a metaanalysis of the epidemiology of angle-closure glaucoma (ACG), to highlight the major demographic risk factors for the development of the disease. This included increasing age (from age 40), female sex, and Asian ethnicity. There was, however, no consideration of socioeconomic deprivation (SED) within the risk factors. 
The adverse effect of SED on health and mortality is well recognised [5]. Studies in both Scotland and England have shown a relationship between increased severity of glaucoma at presentation and greater SED $[6,7]$. SED may affect glaucoma awareness, eye care utilisation, healthcare seeking behaviour and therefore delay identification of asymptomatic glaucoma [8, 9]. It has been suggested that these barriers have become more significant during the COVID pandemic, having implications for the long-term health of patients living in greatest deprivation [10].

Our current practice remains based on the 2015 SIGN guidance [4], which suggests referral to secondary eye care services of all patients found to be at risk of angle closure. This is defined as: (1) using Van Herick technique, if the peripheral anterior chamber width is one quarter or less of the corneal thickness, or (2) using gonioscopy, if $\geq 270$ degrees of posterior pigmented trabecular meshwork is not visible. Patients are then reviewed in clinic, undergo gonioscopy and are categorised as follows: (1) primary angle-closure suspect $(\mathrm{PACS}) \geq 180$ degrees of iridotrabecular contact without raised intra-ocular pressure (IOP) or optic nerve damage: (2) primary angle closure (PAC) $\geq 180$ degrees of irido-trabecular contact with raised IOP but without optic nerve damage: (3) primary angleclosure glaucoma (PACG) $\geq 180$ degrees of irido-trabecular contact with raised IOP and optic nerve damage [11]. We then discuss with our patients the options of monitoring, laser peripheral iridotomy (LPI) or cataract surgery to reduce the risk of acute primary angle closure (APAC), angle-closure glaucoma and potential visual reduction.

The recent publication of the ZAP trial [12] found the incidence of angle-closure disease was very low among individuals classified as PACS identified through communitybased screening in a defined population of a particular age group. The study concludes that the benefit of prophylactic LPI is limited and therefore widespread prophylactic LPI for PACS is not recommended. In the current COVID pandemic, this may be highly clinically significant. However, the implications of this study for an elderly Caucasian population are not clear.

The need to limit footfall through secondary eye care services has never been greater. The ZAP trial [12] alludes to the potential of monitoring a large cohort of the angleclosure referrals in the community, only referring those who need urgent treatment or those that have known risk factors for development of APAC. Some of the known risk factor for precipitating APAC are patients who need repeated pupillary dilation e.g. diabetics, patients on certain groups of medications such as anti-depressants and family history of angle-closure glaucoma [13-15]. We hypothesised that patients with greater SED would be at higher risk of presentation to secondary eye care services with APAC and therefore this should be an additional documented risk factor.

\section{Methods}

A 5-year retrospective study was performed on all patients undergoing LPIs due to PACS/PAC/PACG, including both acute and routine referrals. The study took place across two sites: the Princess Alexandra Eye Pavilion, Edinburgh and the Queen Margaret Hospital, Dunfermline. This provided a large sample size and allowed comparison between two locations of different population structure-Edinburgh and Fife representing an urban and suburban population, respectively. In 2019, the estimated population size of the Princess Alexandra Eye Pavilion, NHS Lothian, was 907,580 and the estimated population size of the Queen Margaret Hospital, NHS Fife was 373,550 [16]. Decision for LPI was at the discretion of the responsible clinician across both centres. Laser logbooks were used to identify all patients that underwent LPIs between 01/01/2015 and 01/ $01 / 2020$. Community referral to both sites is via an electronic glaucoma form which requires minimal clinical information specifically refraction, family history and intraocular pressure.

The Scottish Index of Multiple Deprivation (SIMD) [17] $2020 \mathrm{v} 2$ was used to assess socioeconomic status of each patient as indicated by the patient's postcode. The SIMD provides a relative measure of deprivation across 6976 small areas also known as data zones. The SIMD assess deprivation across seven domains including current income, employment, health, education skills and training, geographic access to service, and housing and crime. The postcode of each patient was allocated to a SIMD rank. The SIMD rank ranges from 1 to 6976, 1 being the most deprived. The SIMD decile represents $10 \%$ of a population with a particular level of deprivation.

Patient records and the TRAK clinical database were used to gather further information including the age, sex, acute or routine presentation, diagnosis (PACS/PAC/ PACG), refractive error, phacoemulsification surgery within 2 years of the laser, use of anti-depressants, family history of glaucoma, and presence of diabetes. APAC referrals were defined as patients referred to the hospital eye service in PAC for review urgently within $24 \mathrm{~h}$. Sub-group analysis of differences between routine referrals versus patients who were referred for acute angle-closure attack were performed using a two-sample $t$-test for continuous variables or Chisquared tests for categorical variables. Logistic regression was used to examine factors that are associated with increased odds of presentation with acute angle closure. Parameters found to be significant on univariate regression analyses were further investigated using multivariable 
regression analyses to determine whether the relationships remained. A $p$-value of $<0.05$ was considered to be statistically significant. Data were analysed using $\mathrm{R}$ software (3.5.1, R Foundation for Statistical Computing, AUT, Vienna, Austria).

\section{Results}

Over the 5-year period at the Princess Alexandra Eye Pavilion, Edinburgh, 612 patients underwent LPIs. This comprised of 401 women and 211 men. The mean age of patient was 68.6 years $( \pm 12.0)$. In total 581 bilateral LPIs were performed and 31 unilateral, giving a total of 1193 lasered eyes. Within a follow of up to 2 years, 155 patients (25.3\%) had cataract surgery. The predominant ethnicity was 'White British/Irish', with 403 (65.8\%) patients selfidentifying in this category. However, it must be noted that a total of $202(33.0 \%)$ of the study population refused to give their ethnicity. The UK 2011 [18] census found the City of Edinburgh to have a $91.7 \%$ white population. Indeed, there were only seven individuals that identified as anything other than 'white British/Irish' representing 1\% of both the routine and acute cohorts. The referral was acute for an APAC in $93(15.2 \%)$ cases and the referral was routine for PACS/PAC/PACG in $519(84.8 \%)$ cases. A diagnosis of PAC was given in 106 (17.3\%) individuals, PACG in $120(19.6 \%)$ individuals and PACS in 386 $(63.1 \%)$ individuals. Refractive data were collected from 1071 of 1193 eyes. The average spherical equivalent was $+1.84( \pm 2.5)$. Finally, $76(12.4 \%)$ individuals were noted to have diabetes, $211(34.5 \%)$ had a positive family history of glaucoma and 126 (20.6\%) of patients were using antidepressant medications. Figure 1 shows the number of LPIs performed over the course of 5 years which trended downwards, but this did not achieve statistical significance $(P=0.0913)$. A sub-group analysis investigating differences in the acute and routine referral groups can be seen in Table 1.

Patients presenting to the Princess Alexandra Eye Pavilion, Edinburgh, with APAC had a lower mean SIMD rank and decile than the patients presenting to routine laser clinics for outpatient LPI. This was found to be statistically significant with a $P$-value of 0.028 .

Over the 5-year period at the Queen Margaret Hospital, Dunfermline, 106 patients underwent LPIs. This comprised of 68 women and 38 men. The average age of patient was 72.2 years $( \pm 11.6)$. In total 94 bilateral LPIs were performed and 12 unilateral, giving a total of 200 lasered eyes. Within a follow of up to 2 years, 20 patients $(18.9 \%)$ had cataract surgery. The predominant ethnicity was White British/Irish, with 101 (96.2\%) patients falling into this category. This is in keeping with the 2011 UK census [18],
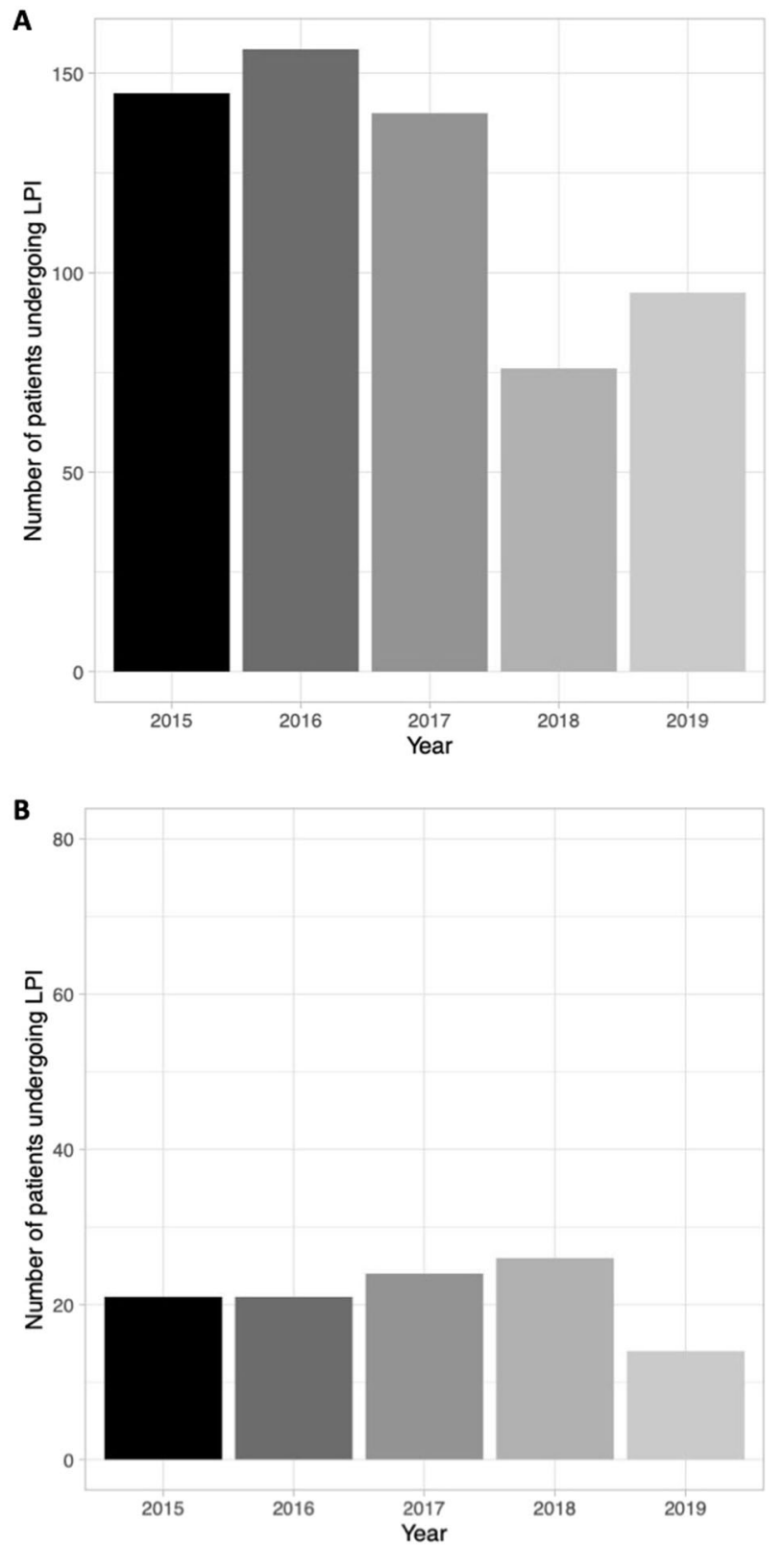

Fig. 1 Number of patients who underwent LPIs between 2015 and 2019. A Princess Alexandra Eye Pavilion, Edinburgh and B Queen Margaret Hospital, Fife.

which stated that the white population within Fife was 97.6\%. The referral was acute for APAC in $26(24.5 \%)$ cases and the referral was routine for PACS/PAC/PACG in $80(75.5 \%)$ cases. A diagnosis of PAC was given in $10(9.4 \%)$ individuals, PACG in $31(29.2 \%)$ individuals and PACS in $65(61.3 \%)$ individuals. Refractive data was collected from 162 of 200 eyes. The average spherical equivalent was $+1.71( \pm 2.8)$. Finally, $12(12.6 \%)$ patients were noted to have diabetes, $17(16.0 \%)$ had a positive family history of glaucoma and $5(4.7 \%)$ of patients were using antidepressant medications. 
Table 1 Demographics of patients who attended the Princess Alexandra Eye Pavilion for LPIs in 2015-2019 categorised by type of referral.

\begin{tabular}{|c|c|c|c|}
\hline Characteristic & $\begin{array}{l}\text { Routine referral, } \\
(N=519)\end{array}$ & $\begin{array}{l}\text { Acute primary } \\
\text { angle closure, } \\
(N=93)\end{array}$ & $P$-value \\
\hline $\operatorname{Sex}(F)$ & $340(65.5 \%)$ & $61(65.6 \%)$ & 0.988 \\
\hline Age (years) & $64.7(12.2)$ & $68.6(9.5)$ & $<0.001$ \\
\hline Ethnicity & & & 0.893 \\
\hline $\begin{array}{l}\text { White British/ } \\
\text { Irish }\end{array}$ & $339(65.3 \%)$ & $64(68.9 \%)$ & \\
\hline Asian & $5(1.0 \%)$ & $1(1.1 \%)$ & \\
\hline $\begin{array}{l}\text { Mixed or } \\
\text { multiple } \\
\text { ethnicities }\end{array}$ & $1(0.2 \%)$ & $0(0.0 \%)$ & \\
\hline Unknown & $174(33.5 \%)$ & $28(30.1 \%)$ & \\
\hline Spherical equivalent & $+1.87(2.51)$ & $+1.85(2.32)$ & 0.970 \\
\hline $\begin{array}{l}\text { Cataract extraction } \\
<2 \text { years }\end{array}$ & $88(17.0 \%)$ & $67(72.0 \%)$ & $<0.001$ \\
\hline Coexisting diabetes & $59(11.4 \%)$ & $15(16.3 \%)$ & 0.186 \\
\hline $\begin{array}{l}\text { Positive family } \\
\text { history }\end{array}$ & $148(31.0 \%)$ & $9(11.1 \%)$ & $<0.001$ \\
\hline $\begin{array}{l}\text { Use of anti- } \\
\text { depressants }\end{array}$ & $101(19.5 \%)$ & $25(27.2 \%)$ & 0.096 \\
\hline \multicolumn{4}{|l|}{ Diagnosis } \\
\hline PAC & $63(12.1 \%)$ & $39(41.9 \%)$ & \\
\hline PACG & $70(13.5 \%)$ & $50(53.8 \%)$ & \\
\hline PACS & $386(74.4 \%)$ & $4(4.3 \%)$ & \\
\hline SIMD Rank & 4397.0 (1952.5) & 3885.5 (2065.4) & 0.028 \\
\hline SIMD Decile & $6.7(2.8)$ & $6.0(3.0)$ & 0.025 \\
\hline
\end{tabular}

Numbers represent mean (SD) or number (\%) unless stated otherwise.

We performed a sub-group analysis of diabetes, family history and antidepressant use. This can be seen in Table 2 . When analysing the routine and acute cohorts, there were no statistically significant results for diabetes or antidepressant use in either NHS Lothian or NHS Fife. It was noted that a positive family history of glaucoma was more prevalent in the routine NHS Lothian cohort and this was found to be statistically significant with a $p$-value of $<0.001$.

Patients presenting to the Queen Margaret Hospital, Dunfermline, with APAC were more likely to have a lower SIMD rank and decile and therefore came from more socially deprived areas than the patients presenting to routine laser clinics for outpatient LPI. This was found to be statistically significant with a $P$-value of 0.035 .

On univariate logistic regression, a negative family history of glaucoma $(P \leq 0.001)$, increasing age $(P=0.019)$ and decreasing SIMD decile $(<0.001)$ were found to be associated with APAC as shown in Table 3. These associations remained significant (all $P<0.01$ ) when examined using multivariable logistic regression (Table 4).
Table 2 Demographics of patients who attended the Queen Elizabeth Hospital for LPIs in 2015-2019 categorised by type of referral.

\begin{tabular}{lccr}
\hline Characteristic & $\begin{array}{l}\text { Routine } \\
\text { referral, } \\
(N=80)\end{array}$ & $\begin{array}{l}\text { Acute primary angle- } \begin{array}{l}P- \\
\text { closure attack, } \\
(N=26)\end{array} \\
\text { value }\end{array}$ \\
\hline $\begin{array}{l}\text { Sex (F) } \\
\text { Age (years) }\end{array}$ & $53(66.3 \%)$ & $15(57.7 \%)$ & 0.429 \\
Ethnicity & $69.9(9.8)$ & $66.6(15.2)$ & 0.304 \\
$\quad$ White British/ & $76(96.2 \%)$ & $25(96.2 \%)$ & 0.511 \\
$\quad$ Irish & $2(2.5 \%)$ & $0(0.0 \%)$ & \\
$\quad$ Asian & $0(0 \%)$ & $0(0 \%)$ & \\
$\quad \begin{array}{l}\text { Mixed or } \\
\text { multiple } \\
\quad \text { ethnicities }\end{array}$ & $1(1.3 \%)$ & $1(3.8 \%)$ & \\
$\quad$ Unknown & & & \\
Spherical equivalent & $+2.1(2.4)$ & $+1.7(3.1)$ & 0.177 \\
Cataract extraction & $9(11.3 \%)$ & $11(42.3 \%)$ & $<0.001$ \\
$<2$ years & & & \\
Coexisting diabetes & $7(9.9 \%)$ & $5(20.8 \%)$ & 0.162 \\
$\begin{array}{l}\text { Positive family } \\
\text { history }\end{array}$ & $13(24.1 \%)$ & $4(21.1 \%)$ & 0.789 \\
Use of anti- \\
depressants
\end{tabular}

Numbers represent mean (SD) or number (\%) unless stated otherwise.

Table 3 Univariate logistic regression examines the relationship between known risk factors of acute angle-closure glaucoma and its presentation.

\begin{tabular}{lrlr}
\hline Factor & Odds ratio & $95 \%$ CI & $P$-value \\
\hline Anti-depressants & 0.345 & -0.149 to 0.814 & 0.159 \\
Family history of glaucoma & -1.069 & -1.723 to -0.491 & $<0.001$ \\
Diabetes & 0.500 & -0.067 to 1.029 & 0.073 \\
Age & 0.020 & 0.003 to 0.038 & 0.019 \\
Sex & 0.076 & -0.340 to 0.482 & 0.715 \\
Spherical Equivalent & -0.011 & -0.101 to 0.079 & 0.804 \\
SIMD Decile & -0.116 & -0.186 to -0.048 & $<0.001$ \\
\hline
\end{tabular}

\section{Discussion}

This study found that patients presenting to both NHS Lothian and NHS Fife with APAC were from a lower rank of the SIMD and therefore greater SED, when compared to the population attending for routine LPI. This is the largest study investigating the importance of socioeconomic status 
Table 4 Results of multivariable logistic regression analyses examining the relationship between factors identified to be significantly associated with the acute presentation of acute angle closure.

\begin{tabular}{lrcr}
\hline Factor & Odds ratio & $95 \%$ CI & $P$-value \\
\hline SIMD Decile & -0.101 & -0.178 to -0.026 & 0.008 \\
Age & 0.029 & 0.009 to 0.049 & 0.004 \\
Family history of glaucoma & -1.010 & -1.670 to -0.426 & 0.001 \\
\hline
\end{tabular}

as a risk factor for APAC in the western world. In alignment with the 2015 SIGN glaucoma guidelines [4] we found that hypermetropia and female sex were risk factors for angle closure, but they did not have a statistical impact on the development of APAC. A positive family history was noted to be more prevalent in the routine referral group within NHS Lothian, perhaps influencing the optometrist's decision to initiate the referral in the first place. The use of antidepressants was noted to be higher in the APAC group, but again this was not statistically significant.

The results of our study, despite being eleven years on, echo the research conducted by Professor Peter Shah et al. in 2009 [19], which reported that patients with acute primary angle closure were more likely to come from areas with higher levels of social deprivation in England. In their study, the Index of Multiple Deprivation 2004 and Townsend score were used to investigate social deprivation, which is largely comparable to the SIMD index.

We acknowledge that SIMD is an area-based measure of deprivation and therefore not every person in a highly deprived area will be experiencing high levels of deprivation. Sloggett et al. [20] reported that 55\% of the most deprived individuals in England and Wales live outside the $20 \%$ most deprived areas. Lack of deprivation does not necessarily equate with increased affluence as the income domain of the SIMD is not a measure of average salary but the number of individuals within an area receiving income support [21]. However, the SIMD has been specifically validated for the Scottish population and remains the gold standard for classification of deprivation within this population.

The major difference between our study and the study conducted by Professor Shah et al. in Birmingham [19], is our population. Neither the city of Edinburgh nor the district of Fife are ethnically diverse populations, particularly when compared to Birmingham, as shown by the 2011 UK census [18]. There was no statistical difference found in the ethnicity of the routine or acute cohorts in either study population, as the numbers of individuals identifying as anything other than white British/Irish were so low. This suggests that there must be alternate factors and potential healthcare barriers creating the relationship between SED and APAC.
First, we must accept that there is a difference in health seeking behaviour between areas of differing SED. Scotland is the only country where free universal NHS funded eye testing is available for all ages. Although this government policy has resulted in more people attending regular comprehensive eye examinations, there is evidence to suggest that there is variable engagement with the service across the socioeconomic spectrum. This facility has been underutilised by people from greater deprivation [22] suggesting that inequalities in eye care service utilisation have increased since the free eye care policy was introduced. Naturally one postulates whether a geographic barrier to healthcare access is present. A 2017 study [23] looking at the distribution of optometric practices relative to deprivation index in Scotland noted that practices were distributed evenly across socioeconomic areas. The suggestion being that differences in eye-examination uptake across social strata are unrelated to service availability.

Poorer utilisation of healthcare services naturally leads to delayed presentation and more advanced disease. This has been shown to be the case in many conditions including skin, breast, and colorectal cancer [24-26] and also a lower uptake of screening programmes such as mammography and cervical screening [27]. The rate of cataract surgery in Scotland, along with the rate of LPI has risen in recent decades. This has reduced the overall rate of APAC [28]. However, the development of cataract remains a major factor in the anatomical development of angle closure and increased prevalence of cataract, in part related to delayed healthcare access, among higher SED is well known [29], predisposing these individuals to APAC.

Both poor literacy rate and education have been shown to negatively affect people's engagement with ophthalmic assessment and treatement [30]. Lower levels of education and literacy are factored into the SIMD calculation and are associated with greater SED, creating a further barrier to healthcare seeking behaviour. Interestingly despite hypermetropia being associated with both PACG and lower educational attainment [31], we did not find a higher level of hypermetropia within our APAC patients.

A 2016 nationwide Taiwanese population based study [32] found that patients diagnosed with PACG were more likely to come from greater SED whereas those diagnosed with POAG were more likely to have less SED. In contrast to our study, the findings in Taiwan were felt to be due to an increased anatomical susceptibility to PACG rather than SED causing a barrier to healthcare access. The increased susceptibility was postulated to be related to less myopia and shorter stature in patients from a more deprived background, both of which are well known risk factors for PACG [33].

We performed a sub-group analysis of all our patients presenting with PACG and found no statistically significant 
data to suggest they were from greater SED. The similar results obtained in Western and Asian countries is interesting as it highlights the importance of SED as a risk factor irrespective of ethnicity, which is classically identified as a risk factor for primary angle-closure glaucoma.

Best practice regarding secondary eye care assessment of all patients with PACS/PACG/PAC remains undecided. Sadly, widening healthcare inequality is an anticipated consequence of the COVID pandemic. The combination of postponed outpatient attendances and delays in, or avoidance of, accessing healthcare is anticipated to have longterm adverse impact for people living in the most deprived areas [10]. Prior to the COVID pandemic the Scottish government backed increasing use of community optometry services and there is growing recognition that appropriately trained independent prescribing optometrists can provide traditionally hospital-based services in the community [34]. It is likely that through the pandemic and in a post-pandemic climate there will be increased collaboration between independent optometry providers and hospital services. When considering risk stratification for new referrals for hospital assessment, we propose that patients' level of SED should be considered as a risk factor for future acute angle-closure attack. Therefore, a heightened awareness when processing patients from deprived areas and considering threshold for LPI and cataract surgery is appropriate.

In conclusion, this is the first Scottish study to investigate the relationship between socioeconomic deprivation and angle-closure disease. Due to the limited differences in ethnicity, sex, refraction, and past medical history between the two cohorts within our study, we suggest that the relationship between SED and presentation with APAC is primarily due to barriers related to healthcare utilisation. We believe socioeconomic deprivation is an important risk factor for patients progressing from PACS to APAC. Although there is presently no nationally agreed glaucoma risk stratification model, we would propose that in addition to the long-recognised patient demographic factors, socioeconomic status should be considered in decision making tools.

Further work validating individualised risk assessments that incorporate rate of disease progression, visual needs, and demographic factors is urgently needed to assist in the rationalisation of NHS services, including LPI and cataract extraction, during the pandemic and to protect the vision of glaucoma patients in the post-pandemic years to come.

\section{Summary}

\section{What was known before}

- Socioeconomic deprivation is associated with lower life expectancy and less interaction with healthcare services.
- Socioeconomic deprivation is associated with more advanced presentation of glaucoma in Scotland.

\section{What this study adds}

- Higher levels of socioeconomic deprivation are a risk factor for presentation to ophthalmic services with acute primary angle closure.

Author contributions ES was responsible for data collection, extracting and analysing data, interpreting results, and writing the report. $\mathrm{KC}$ was responsible for data collection, extracting and analysing data, interpreting results, and assisting in the writing of the report. NOC was responsible for data collection, extracting and analysing data, interpreting results, and assisting in the writing of the report. RS provided expert advice and guidance on the construction of the study and provided feedback on the report. PKA devised the idea of the project. He provided expert advice and guidance on the construction of the study and provided feedback on the report.

\section{Compliance with ethical standards}

Conflict of interest The authors declare no competing interests.

Publisher's note Springer Nature remains neutral with regard to jurisdictional claims in published maps and institutional affiliations.

\section{References}

1. Quigley HA, Broman AT. The number of people with glaucoma worldwide in 2010 and 2020. Br J Ophthalmol. 2006;90:262-7.

2. Bunce C, Wormald R. Leading causes of certification for blindness and partial sight in England \& Wales. BMC Public Health. 2006;6:58.

3. Jayaram H, Strouthidis NG, Gazzard G. The COVID-19 pandemic will redefine the future delivery of glaucoma care. Eye Lond Engl. 2020;34:1203-5.

4. Scottish Intercollegiate Guidelines Network (SIGN). Glaucoma Referral and Safe Discharge: A National Clinical Guideline. SIGN; 2015.

5. Smith GD, Carroll D, Rankin S, Rowan D. Socioeconomic differentials in mortality: evidence from Glasgow graveyards. BMJ. 1992;305:1554-7.

6. Ng WS, Agarwal PK, Sidiki S, McKay L, Townend J, AzuaraBlanco A. The effect of socio-economic deprivation on severity of glaucoma at presentation. Br J Ophthalmol 2010;94:85-7.

7. Fraser S, Bunce C, Wormald R, Brunner E. Deprivation and late presentation of glaucoma: case-control study. BMJ. 2001;322:639-43.

8. Hoevenaars JGMM, Schouten JSAG, van den Borne B, Beckers HJM, Webers CAB. Socioeconomic differences in glaucoma patients' knowledge, need for information and expectations of treatments. Acta Ophthalmol Scand. 2006;84:84-91.

9. Zhang X, Beckles GL, Chou CH, Saaddine JB, Wilson R, Lee P, et al. Socioeconomic disparity in use of eye care services among US adults with age-related eye diseases: National Health Interview Survey, 2002 and 2008. JAMA Ophthalmol. 2013;131: 1198-206. 
10. Scottish Government. The Impacts of COVID-19 on Equality in Scotland. Scottish Government; 2020. https://www.gov.scot/bina ries/content/documents/govscot/publications/research-and-ana lysis/2020/09/the-impacts-of-covid-19-on-equality-in-scotland/.

11. Foster PJ, Buhrmann R, Quigley HA, Johnson GJ. The definition and classification of glaucoma in prevalence surveys. Br J Ophthalmol. 2002;86:238-42.

12. He M, Jiang Y, Huang S, Chang D, Munoz B, Aung T, et al. Laser peripheral iridotomy for the prevention of angle closure: a singlecentre, randomised controlled trial. Lancet Lond Engl. 2019;393:1609-18.

13. Amerasinghe N, Aung T. Angle-closure: risk factors, diagnosis and treatment. Prog Brain Res. 2008;173:31-45.

14. Ah-Kee EY, Egong E, Shafi A, Lim LT, Yim JL. A review of druginduced acute angle closure glaucoma for non-ophthalmologists. Qatar Med J. 2015;2015:6.

15. Tan GS, Wong CY, Wong TY, Govindasamy C, Wong E, Yeo I, et al. Is routine pupil dilation safe among asian patients with diabetes? Invest Ophthalmol Vis Sci. 2009;50:4110-3.

16. National Records of Scotland. Mid-2019 Population Estimates Scotland. 2020. https://www.nrscotland.gov.uk/files//statistics/ populationestimates/mid-19/mid-year-pop-est-19-report.pdf.

17. Scottish Government. Scottish Index of Multiple Deprivation. 2020. https://www.gov.scot/collections/scottish-index-of-multipledeprivation-2020/.

18. National Records of Scotland. Scotland's Census. 2011. https://www.scotlandscensus.gov.uk/ods-analyser/jsf/tableView/ta bleView.xhtml.

19. Nessim M, Denniston AK, Nolan W, Holder R, Shah P. Research into Glaucoma and Ethnicity (ReGAE) 8: is there a relationship between social deprivation and acute primary angle closure? Br J Ophthalmol. 2010;94:1304-6.

20. Sloggett A, Joshi H. Higher mortality in deprived areas: community or personal disadvantage? BMJ. 1994;309:1470-4.

21. Stephens MR, Evans M, Ilham MA, Marsden A, Asderakis A. The influence of socioeconomic deprivation on outcomes following renal transplantation in the United kingdom. Am J Transplant. 2010;10:1605-12.

22. Dickey H, Ikenwilo D, Norwood P, Watson V, Zangelidis A. Utilisation of eye-care services: the effect of Scotland's free eye examination policy. Health Policy Amst Neth. 2012;108: 286-93.

23. Legge R, Strang NC, Loffler G. Distribution of optometric practices relative to deprivation index in Scotland. J Public Health. 2018;40:389-96.

24. Wells BL, Horm JW. Stage at diagnosis in breast cancer: race and socioeconomic factors. Am J Public Health. 1992;82:1383-5.

25. Powe BD. Fatalism among elderly African Americans. Effects on colorectal cancer screening. Cancer Nurs. 1995;18:385-92.

26. Robinson JK, Altman JS, Rademaker AW. Socioeconomic status and attitudes of 51 patients with giant basal and squamous cell carcinoma and paired controls. Arch Dermatol 1995;131:428-31.

27. Calle EE, Flanders WD, Thun MJ, Martin LM. Demographic predictors of mammography and Pap smear screening in US women. Am J Public Health. 1993;83:53-60.

28. Gillan SN, Wilson PJ, Knight DS, Sanders R. Trends in acute primary angle-closure glaucoma, peripheral iridotomy and cataract surgery in Scotland, 1998-2012. Ophthalmic Epidemiol. 2016;23:1-5.

29. Keenan T, Rosen P, Yeates D, Goldacre M. Time trends and geographical variation in cataract surgery rates in England: study of surgical workload. Br J Ophthalmol 2007;91:901-4.

30. Muir KW, Santiago-Turla C, Stinnett S, Herndon L, Allingham RR, Challa P, et al. Health literacy and adherence to glaucoma therapy. Am J Ophthalmol 2006;142:223-6.

31. Wong TY, Foster PJ, Johnson GJ, Seah SKL. Education, socioeconomic status, and ocular dimensions in Chinese adults: the Tanjong Pagar Survey. Br J Ophthalmol. 2002;86:963-8.

32. Ko Y-C, Hwang D-K, Chen W-T, Lee C-C, Liu CJ. Impact of socioeconomic status on the diagnosis of primary open-angle glaucoma and primary angle closure glaucoma: A Nationwide Population-Based Study in Taiwan. PloS ONE. 2016;11: e0149698.

33. Boland MV, Zhang L, Broman AT, Jampel HD, Quigley HA. Comparison of optic nerve head topography and visual field in eyes with open-angle and angle-closure glaucoma. Ophthalmology. 2008;115:239-245.e2.

34. Scottish Government. Community Eyecare Services: Review. Scottish Government; 2017. https://www.gov.scot/publications/ community-eyecareservices-review/. 